\title{
De la table, une œuvre d'Habib Tengour en cours de rédaction
}

Présentation de l'inédit ainsi que du dossier génétique de l'œuvre

Hervé Sanson

\section{(2) OpenEdition}

\section{Journals}

Electronic version

URL: http://journals.openedition.org/coma/771

DOI: $10.4000 /$ coma.771

ISSN: 2275-1742

Publisher

Institut des textes \& manuscrits modernes (ITEM)

\section{Electronic reference}

Hervé Sanson, "De la table, une œuvre d'Habib Tengour en cours de rédaction », Continents manuscrits [Online], 7 | 2016, Online since 20 December 2016, connection on 20 April 2019. URL :

http://journals.openedition.org/coma/771 ; DOI : 10.4000/coma.771

This text was automatically generated on 20 April 2019.

\section{(c)}

Continents manuscrits - Génétique des textes littéraires - Afrique, Caraîbe, dispora est mis à disposition selon les termes de la licence Creative Commons Attribution - Pas d'Utilisation Commerciale - Pas de Modification 4.0 International. 


\title{
De la table, une œuvre d'Habib Tengour en cours de rédaction
}

\author{
Présentation de l'inédit ainsi que du dossier génétique de l'œuvre
}

\author{
Hervé Sanson
}

1 Il est rare d'entrer dans le processus de genèse de l'œuvre lorsque cette œuvre n'a pas encore trouvé son terme. Le plus souvent, le généticien se penche sur l'ensemble des avant-textes, bien après la publication du roman, du poème, de la pièce de théâtre. C'est dire la confiance qu'Habib Tengour a su nous accorder, en nous permettant de scruter les « arrière-cours » de la création, et qui plus est, de les publier.

2 Ce dossier génétique est constitué de sept avant-textes, se déployant sur une durée de onze ans. La première date inscrite est le 5 mars 2005 ; en fait ce projet constitue « Le Livre » pour Tengour (titre que les trois premières ébauches inscrivent), et son exécution fut maintes fois repoussée avant que l'écrivain ne se décide à sauter le pas et à rédiger un premier synopsis de ce qu'il conviendrait de nommer une épopée. Le lecteur adepte de l'œuvre de Tengour retrouve là le mode opératoire propre au poète: la reprise sur le métier maintes et maintes fois d'un même texte, du premier brouillon à la publication en revue, puis à la reprise en recueil.

3 Les différentes étapes indiquées par cet avant-projet obéissent à un ordre chronologique : depuis l'instauration de l'état civil pour les Algériens dans la seconde moitié du XIX siècle jusqu'aux années 1990, années du retour en France pour Tengour. Ce synopsis montre d'emblée le souci de l'écrivain de tresser l'histoire tribale, puis personnelle, avec la grande Histoire afin d'attester de la présence de certains événements, c'est-à-dire de témoigner, en transmettant une mémoire aux générations suivantes.

4 Tengour insiste sur le caractère non exhaustif de son projet, et sur la dimension compositionnelle que l'œuvre doit endosser, c'est-à-dire sur sa capacité à recréer les faits rapportés en les nommant (l'un des avant-textes porte pour titre « Le Roman du nom »). Autrement dit : comment raconter? Quelle structure adopter? Plusieurs des avant-textes ici publiés font état de cette préoccupation majeure de l'écrivain. Ainsi les trois états intitulés « Le Livre » portent-ils cette injonction que l'écrivain se fait à lui-même : « Tout ça demande à être construit. » 
5 Dès le second avant-texte nommé «Le Livre ", un ajout, daté du 4 août 2008, évoque l'anecdote de la table gigantesque sur laquelle les officiers français procédèrent à l'enregistrement des "indigènes » à l'état civil, et notamment des tribus auxquelles le futur écrivain Habib Tengour est affilié. Le reste des avant-textes composant le dossier génétique proposé au lecteur n'est que l'ébauche, puis le développement de cette séquence initiale, d'abord nommée "La Table », puis "De la table». À cette vision inaugurale de la table qui signe une nouvelle ère pour les tribus pacifiées, s'associe la peinture de la tribu en sa magnificence, et de son passé glorieux.

6 L'inédit proposé ici au lecteur est organisé autour du motif de cette table, métonymie d'un basculement des temps pour les colonisés. Les familiers de l'œuvre d'Habib Tengour pourront tracer telle ou telle analogie, opérer tel ou tel rapprochement avec certaines de ses œuvres, telles Gens de Mosta (1997) ou bien Le Maître de l'heure (2008).

7 Je publierai dans un prochain numéro de Continents manuscrits une étude approfondie sur ce dossier génétique, lequel sera entre-temps étoffé d'autres éléments, aussi bien tapuscrits que manuscrits.

Inédit : De la table

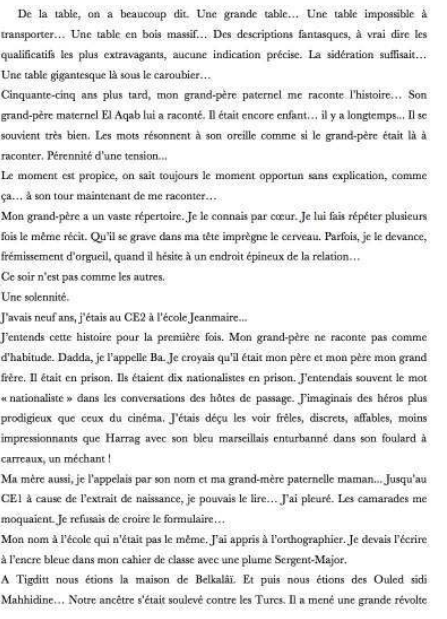




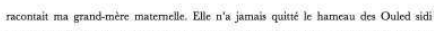
Mathidine

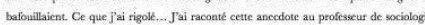

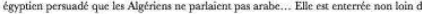
masiolece de Sid Ben Chat. Une tombe anonyme amas de pierres sans stele un bol cen

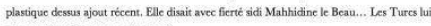
ont coupe la ta tiec comme i Habrchi

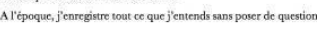

La voix de grand-pere n'est pas la meme pour raconter I'tepopte du roi Seir ibs

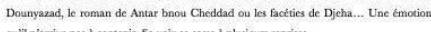

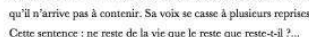

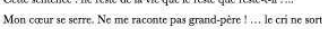

Jattends.

Je ne dis rien. Je ne sais pas.

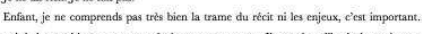

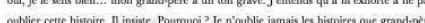

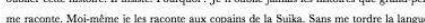

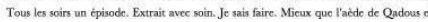

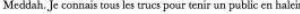

La bande prefête mes histoires a cecles de Kader. Lui, il invente et on n'y croit pas.... gagne le fourbe quand ii raconte le films quil a vus ì Oran. II passe les vacances de lée chere son onde matemel a sid el Houari. A Mosta, on doit artendre un an pour les vonty

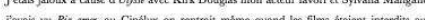
petich je

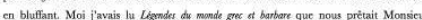
Marin. Je connaissais mícux Phissoire d'Uyyse et la guecre de Troie que Kader. II te celui qui racontait, sur qui repose le film? ?... Reppondre ou etre diaqualiféc..II appelait Ulysect le cyclope

II y a des films,je se sis sur, illes invertait

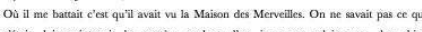

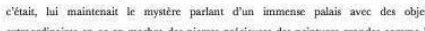

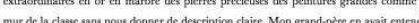

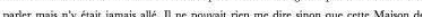

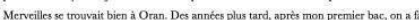

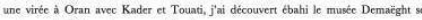
sculpturss de Beclmondo et un pecit Foujtita.

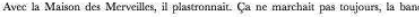

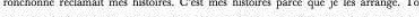

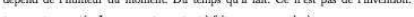

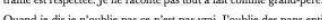

medper me

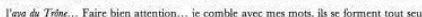
je ne les invente pas. Ils simbriquent naturclenent avec les autres dans la narration. Les copains ne remargunat rien... 


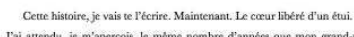

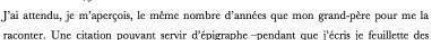

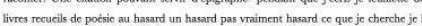

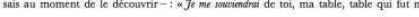

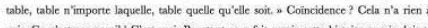

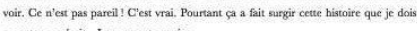
taconter par écrit.. Le moment propice.

Jefer

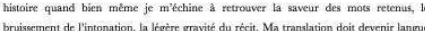
originale. Question de vic ou de mor. Je n'ai pas le choo

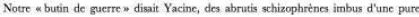

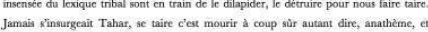
riquere la mort Un butin a du sens si ion le frconde.

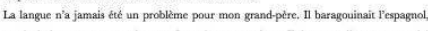
tenait de longue conversations en fançusis avec madame Delage, sa cliente au march des linguese... Pourtant analphabete

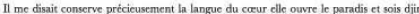

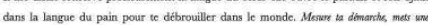

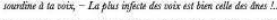

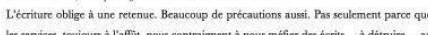

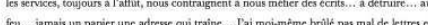
d'crits pa per ber

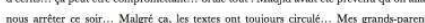
auss briliaient las lives les papiess les photos de mon pere avant perquisition... $\mathrm{Ce}$ autodalfs me hanter

La viox peut sétranghte

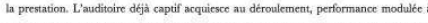

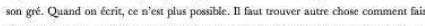

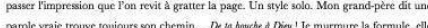
parole vaic roun a jo

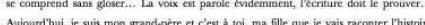

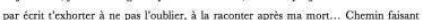

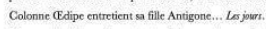

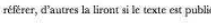

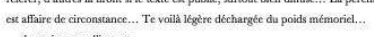

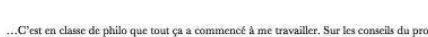

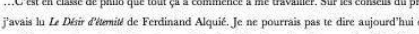

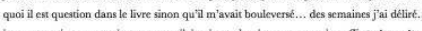

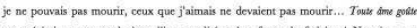

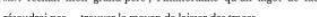

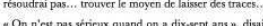

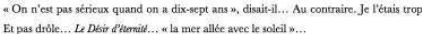

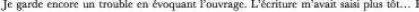

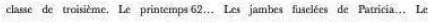
Romantiques au programme... La protparation du BEFC sur les bancs des Bur

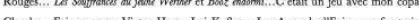

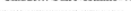
d'une montec au maq is

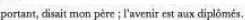

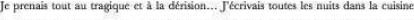

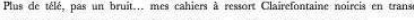

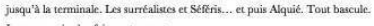

Je ne pouvais plus fairc autrement 


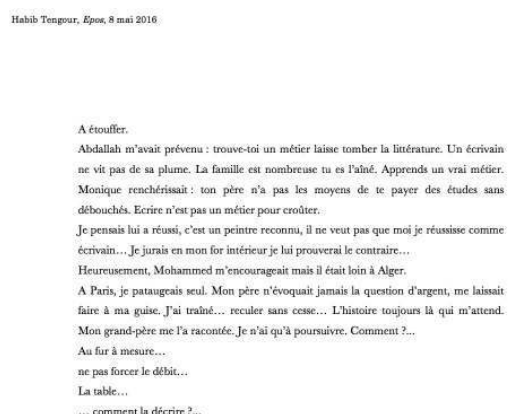

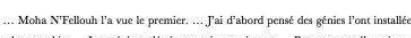
sous le caroutiec... Las genies, étaiti pour impressionect... Personne ne la pris an serieux. Les Ouled Sid Ahmed II ne plaisantait pas II avait bien vu la table sous le caroubier. A quelques mèrese. Une

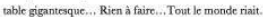
Mon grand-pere ausis ina

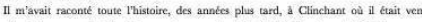

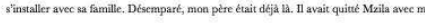
merere et ma scuur Hlima pour venir a LHilli... A cause de toute Phistoire... Cess tiq que

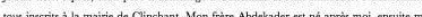

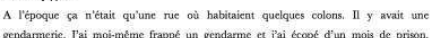

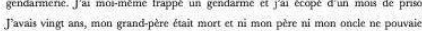

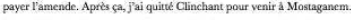

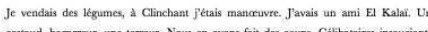

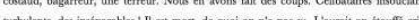
contiaguc a

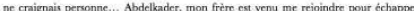
larmece. If a tete embauche comme docker au port. A cette epoque, on a changet transcription du nom de 'létat civil. Cest bien plus tard.

Lhistoire que je raconte sc passe avant.

Habib Tengour 
Dossier génétique : Le Livre 2

\begin{abstract}
Le Kremlin, samedi 5 mars 2005
Ce live qui attend depuis Päge de dix-sept ans. II est peut-tere temps de s' accrocher. Jen parle en toute occasion, que ça en devient louche. Cette peur de reculer le moment. De toute facon, je ne peux pas y cchapper. Tenter, i réussite, si elle est indispensable, n'est pas obligatoire. Cap prendra quelques anneces mais au fond ça n'est pas plus mal. Je vals pouvoir mettre à profit ce que 'ải appris dans mon bricolage et réfléchir sur ce qui me tient à ccrur, ce «désir d'étemité $n$, la mémoire et la transmission, la restitution du récl par l'art, les trames de la narration, le poème, etc. Un texte qui concentre un peuple, un groupe, une nation pour s'ouviri à tous, línstant et le temps, Pespace...

If faut trouver la forme, ar le vrai problème est formel.

Lhistoire est simple, le exexte doit Pêtre. Ellle se déroule depuis la fin du XIX' siècle Guste après la mise en place des lois agraires: Loi Wamier ef Sénatus-consulte), au moment de linstauration de P'état civil. Elle commence à Mzila, chea les Beni Zeroual de la plaine du Chelif avec l'éparpillement de

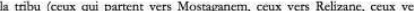
Orane, pus tand l'émigration vers b. France, le retour dans le pays indépendant, ,... jusqu'à aujourd'hui

La première partic : Le nom. Les burcaux arabes procódent à l'opéntion l'écteblissement de l'ótat civil Ils vont donner des noms de famille aux

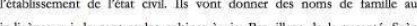
indigenes qui devront se les coltinet a vie. Broullage de la parentite. Scène nommer. Pétrus Borel. Toun pour toons.
\end{abstract}

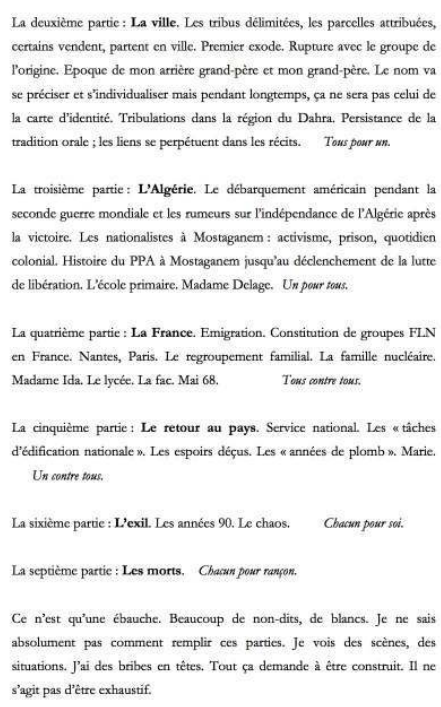

Habib Tengour 


\section{Dossier génétique : Le Livre 1}

Le Kremlin, samedi 5 mars 2005

Ce live qui attend depuis Pagge de dix-sept ans. II est peut-erec temps de s'y accrocher. J'en parle en toute occasion que ça en devient louche. Cette peur de reculer le moment. De toute façon, je ne peux pas y ćchapper. Tenerer, In réussite, si elle est indispensable, n'est pas obligatoire. Cap prendra quelque anneces mais au fond ça n'est pas plus mal. Je vals pouvoir mettre à profit ce que 'ải appris dans mon bricolage et réfléchir sur ce qui me tient à ccrur, co «désir d'éternite $\%$, la mémoire et la transmission, la restitution du récl par lart, les trames de la narration, le poème, etc. Un texte qui concentre un peuple, un groupe, une nation pour s'ouviri à tous, línstant et le temps, Pespace...

If faut trouver la forme, ar le vrai problème est formel.

Lhistoire est simple, le texte doit Pêtre. Ellle se déroule depuis la fin du XIX siècle Guste aptròs la mise en place des lois agraires: Loi Warmier et Sénatus-consulte), au moment de linstauration de P'état civil. Elle commence ì Mzila, chea les Beni Zeroual de la plaine du Chelif avec léparpillement de

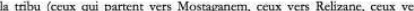

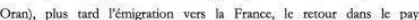
indépendant, ,... jusqu'à aujourd'hui

La premičre partic : Le nom. Les burcaux arabes procódent à l'opération de

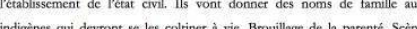
indigenes qui devront se les coltinet a vie. Broullage de la parenté. Scène

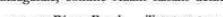

\footnotetext{
La deuxième partic: La ville. Les tribus delimitices, les parcelles atrribuées, certains vendent, partent en ville. Premict exode. Rupture avec le groupe de Porigine. Epoque de mon arrière grand-père et mon grand-père. Le nom ya se préciser et sindividualiser mais pendant longtemps, ca ne sera pas celui de la carte didenentité. Tribulations dans la région du Dahra. Pernistance de la tradition orale ; les liens se perpétuent dans les récits. Touspour

La troisième partie: LAlgérie. Le debarquement américin pendant Seconde Guerre mondiale et les rumeurs sur lindépendance de l'Alpétie après la victoir

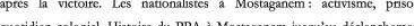
de la lute de liberation. Lécole primaire. Madame Delage. Un peur thases.

La quarticeme partic: La France, Emigration. Constitution de groupes FLN en France. Nantes, Paris. Le regroupement familial. La famille nucleair

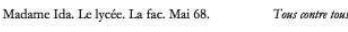

La cinquieme partie: Le retour au pays. Service national. Les « taches d'édification nationale $m$. Les espoirs déçus. Les « années de plomb $m$. Marie Un conte tower:

La sixième partic : Lexil. Les années 90. Le chaos. Chasun pour rsoi.

La sepdième partic : Les morts. Chasun pour rearpon

Ce n'est qu'une ébauche. Beaucoup de non-dits, de blances. Je ne sais absolument pas comment remplir ces partics. Je vois des scènes, des situations. Jai des bribes en têtes. Tout ça demande à è tute construit. Il ne
} s'agit pas d'etre exhausifi. 
Barbârre, le 4 août 2008

La table était gigantesque. Elle avait tété plaće sous le grand caroubie

des Beni Zeroual. Deux officiers siégeaient devant des piles de

formulaires. Quatre soldats montaient la garde. L'aube commençait a

pointer. La journée s'annonçait caniculaire.

Habib Tengour

Dossier génétique : Le Livre, 13 septembre 2007

Le Kremlin, samedi 5 mars 2005

Ce live qui attend depuis Pầge de dix-sept ans. Il est peut-etre temps de s'y accrocher. Jen parle en toute occasion que ca en devient louche. Cette pew de reculer le moment. De toute façon, je ne peux pas y ćchapper. Tenter, la réussite, si elle est indispenseble, p'est pas obligatoire Ca prenda qued années mais au fond ca n'est pas plus mal. Je vais pouvoir mettre ì profit

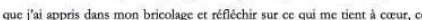
This disto The les mes de b a

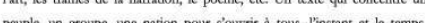
whe respace...

II faut trouver la forme, car le vraj problème est formel

Lhistore est simple, te texte doit Pêtre. Elle se déroule depuis la fin du XIX sieccle Guste après la mise en place des lois agraires: Loi Wamier et Senatus-consulte), au moment de linstauration de Rtat civi. Elle commence ì Mzila, chez les Beni Zeroual de la plaine du Chelif avec l'éparpillement de la tribu (ceux qui partent vers Mostaganem, caux ves Relizanc, ecux vers Oran), plus tard Pémigration vers la France, le retour dans le pays indépendant, ... jusqu'à aujourd"hui

La première partic : Le nom. Les burcaux arabes procódent à l'opéntion de l'ćtablissement de l'ćtat civil. Ils vont donner des noms de famille aux indigènes qui devront se les coltiner à vie. Brouillage de la parenté. Scène inaugurale, comme Adam faisant défler les créatures devant lui pour les nommer. Pétrus Borel. … $\rightarrow$ Teur pour touc 
La deuxième partic: L La ville. Les tribus delimitées, les parcelles attribuces, Porigine. Epoque de mon arričre grand-père et mon grand-père. Le nom và se préciser et sindividualiser mais, pendant longremps, a ne sera pas celui de la carte dididentité Tribulations dans la tónion du Dahra Persistonee de

La trosisieme partie: LAlgetrie. Le debarquement américain pendant ta

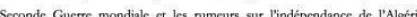

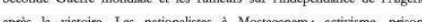

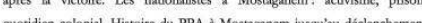
de la lutte de libération. Le bruit de la mer. L'ceole primaire. Le Cinelux. Me la lutte de liberazaion. Ie bruit de la mer. Lecole primaire. Le

La quartième partie : La France. Emigration. Constitution de groupes FLN en France. Nantes, Paris. Le regroupement familial. La famille nucleare Madame Ida. Le lycéé. Le père, La telévision. La fac. L'ével au politique et

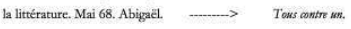

La cinquième partic : Le retour au pays. Service national. Les « táches d'dification nationale m. Les espoirs déçus. Les a annéces de plomb w. Les coopérants. Maric. Les révoltes urbaines. $\quad \longrightarrow \quad \longrightarrow$ Un conhrin touses.

La sixième partic: L'exil. Les années 90. Le chaos. Léclatement de ha famille. Vieilitir ailleur-lì Samre $\longrightarrow$

La septième partic : Les morts. La narration et le chant à transmettre. Le silence du lac. $\quad \longrightarrow$ Chacun pour rangon.

Ce n'est qưune ébauche. Beaucoup de non-dirs, de blancs. Je ne sies absolument pas colvent andit

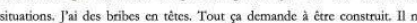

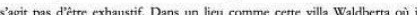
: reprends ces tignes, favancerail beaucoup; muss le lieu importe beacou

Villa Waldberta, le mercredi 25 mai 2005

Habib Tengour 
Dossier génétique : La Table

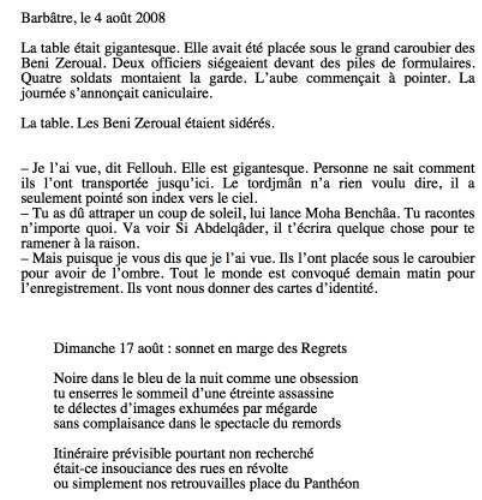

Habib Tengour

Dossier génétique : Le Roman du nom

I. La Table

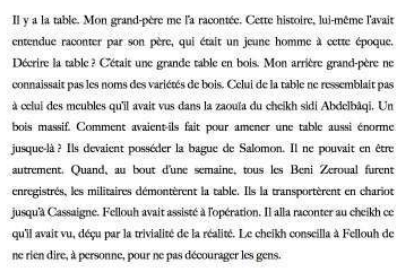

Habib Tengour 
Dossier génétique : Le Roman du cinquantenaire

La table, Les Beni Zeroual n'avaient jamais vu unc table aussi grande. Un bois massiff, qưuls ne connaissaient pas. Ces mécréents pactisient sans aucun doute avec Satan, le lapidé $!$ A moins qư'ls n'aient volé la bague de Salomon pour obliger les dijnns à déplacer une telle table. Mon grand-père el Aqab, qui avait plusieurs fois rendu visite au sheikh sidi Abdelbaqi reconnut que cette table teait au moins dix fois plus grande que celle du maitre. J'teais un petit enfant de six ans et je ne me souviens plus de Pagigtation de cette ípoque. Nous n'avions jamais quitté notre hameau des Ouled sidid Mahiedine et notre connaissance du monde étaiat limitée aux nouvelles que nous rapportait notre maitre, une fois l'an ou les tares fideles quil lui rendaient viste pour une question importante. Cette table installíc sous le caroubier, au bord du sentier qui menait a la rivière relevait du prodige. Un beau matin, Fellouh ameuta les hameaux des Beni Zeroual pour leur siggaler P'événement. Personne n'avait vu l'installation de la able. Les miltaiares ćtraient assis sur des thbourets, seul l'offficer siégeait sur un fauteuil. Sur la table, des piles de dossiers comme les tentes disperséces d'un campenent A un pied de la table, à côté du fauteuil, était posée une carte d'état-major. Nous étions loin de nous douter que ce bout de papier contenait toutes nos unese et nos habitations. Sur un simple coup d'cril, roffficier pouvait situer chacun d'entre-nous avec précision. Jusqu'aux lieux-dits, connus des seculs natifs, étaient d'entre

Habib Tengour

Dossier génétique : La Table, 31 janvier 2015

L.La Table

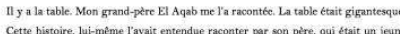

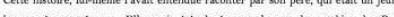

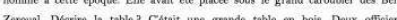
siegeseient devant des piles de formularires. Quarre soldats montaient la gavide

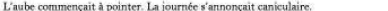
Mon arriere grand-pere est alle la voir. In ne connaissait pas les noms des varietét

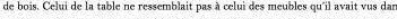

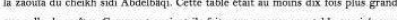

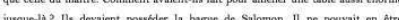

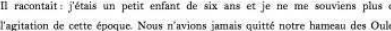
sidid Mahiedine et notre connaissance du monde tatit linitite aux nouvelles que mous rapportait notre maitre, une fois lan ou les rares fiddeses quil tui rendaich

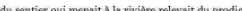

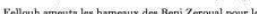

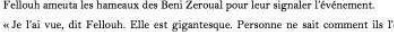

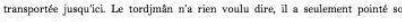
index vers le cicl.

"Tiu as do attraper un coup de soletil, luil lance Moha Benchia. Tu racontes

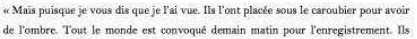

vont nous donner des cartes didentité 


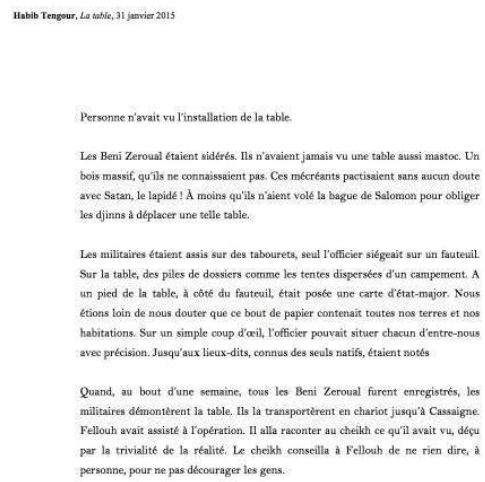

INDEX

Mots-clés: Habib Tengour

\section{AUTHOR}

\section{HERVÉ SANSON}

Rédacteur en chef de la revue Continents Manuscrits, spécialiste de génétique et de la littérature algérienne francophone fr 